\title{
Natural Boundary Conditions for Smoothing in Geometry Processing
}

\author{
ODED STEIN and EITAN GRINSPUN, Columbia University \\ MAX WARDETZKY, Universität Göttingen \\ ALEC JACOBSON, ETH Zurich, Columbia University, and University of Toronto
}

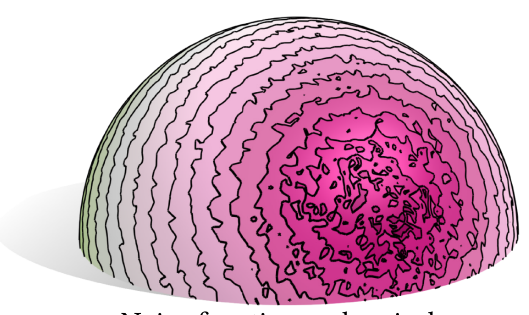

Noisy function on hemisphere

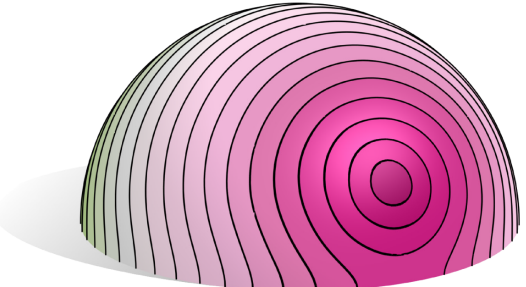

Low-order boundary conditions

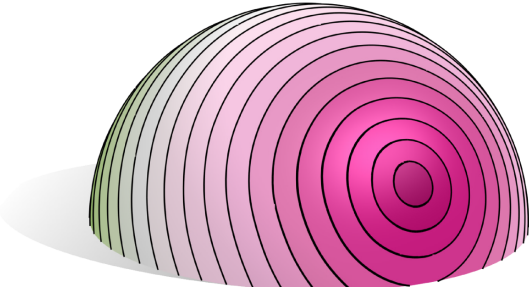

Natural boundary conditions

Fig. 1. Smoothing a noisy function with the Laplacian energy and common low-order boundary conditions (zero Neumann) introduces a bias at the boundary: isolines exit perpendicularly. We propose using a different smoothness energy, the Hessian energy, whose natural boundary conditions avoid this bias.

In geometry processing, smoothness energies are commonly used to model scattered data interpolation, dense data denoising, and regularization during shape optimization. The squared Laplacian energy is a popular choice of energy and has a corresponding standard implementation: squaring the discrete Laplacian matrix. For compact domains, when values along the boundary are not known in advance, this construction bakes in low-order boundary conditions. This causes the geometric shape of the boundary to strongly bias the solution. For many applications, this is undesirable. Instead, we propose using the squared Frobenius norm of the Hessian as a smoothness energy. Unlike the squared Laplacian energy, this energy's natural boundary conditions (those that best minimize the energy) correspond to meaningful high-order boundary conditions. These boundary conditions model free boundaries where the shape of the boundary should not bias the solution locally. Our analysis begins in the smooth setting and concludes with discretizations using finite-differences on 2D grids or mixed finite elements for triangle meshes. We demonstrate the core behavior of the squared Hessian as a smoothness energy for various tasks.

CCS Concepts: - Mathematics of computing $\rightarrow$ Discretization; Partial differential equations; Numerical differentiation; • Computing methodologies $\rightarrow$ Animation; Mesh geometry models;

This work was funded in part by the Binational Science Foundation (US-Israel) Award 2012376, the National Science Foundation Award IIS-14-09286, the NSERC Discovery Grants (RGPIN-2017-05235 \& RGPAS-2017-507938), a Canada Research Chair award, the Connaught Fund, and a gift from Adobe Systems Inc.

Authors' addresses: O. Stein, Columbia University, APAM, 500 W 120th St, Room 200, New York, NY, 10027, USA; email: oded.stein@columbia.edu; E. Grinspun, Columbia University, Computer Science Department, 500 W 120th St, Room 450, New York, NY, 10027, USA; email: eitan@cs.columbia.edu; M. Wardetzky, Universität Göttingen, Institute of Num. and Appl. Math, Lotzestr. 16-18, Göttingen, 37083, Germany; email: wardetzky@math.uni-goettingen.de; A. Jacobson, ETH Zurich, Switzerland, Columbia University, USA, University of Toronto, Bahen Centre for Information Technology, 40 St. George Street, Room 5266, Toronto, ON, M5S 2E4, Canada; email: alecjacobson@gmail.com.

Permission to make digital or hard copies of all or part of this work for personal or classroom use is granted without fee provided that copies are not made or distributed for profit or commercial advantage and that copies bear this notice and the full citation on the first page. Copyrights for components of this work owned by others than ACM must be honored. Abstracting with credit is permitted. To copy otherwise, or republish, to post on servers or to redistribute to lists, requires prior specific permission and/or a fee. Request permissions from permissions@acm.org.

(c) 2018 ACM 0730-0301/2018/04-ART23 $\$ 15.00$

https://doi.org/10.1145/3186564
Additional Key Words and Phrases: Geometry processing, smoothing, denoising, Hessian energy, Laplacian energy

\section{ACM Reference format:}

Oded Stein, Eitan Grinspun, Max Wardetzky, and Alec Jacobson. 2018. Natural Boundary Conditions for Smoothing in Geometry Processing. ACM Trans. Graph. 37, 2, Article 23 (April 2018), 13 pages.

https://doi.org/10.1145/3186564

\section{INTRODUCTION}

Smoothness energies are commonly used in graphics, geometry processing and image processing to model deformations, denoise densely sampled measurements, and interpolate sparse scattered data. Compared to those involving first derivatives, smoothness energies involving second derivatives are by definition more expressive, but also more challenging to control. For example, minimizing the squared-norm of the gradient of an unknown function $u$ integrated over a bounded domain $\Omega \subset \mathbb{R}^{2}$,

$$
E_{\nabla^{2}}(u)=\frac{1}{2} \int_{\Omega}\|\nabla u\|^{2} d A,
$$

results in a second-order Laplace equation with only one set of prescribable boundary conditions (either fixed values, normal derivatives, or linear combinations thereof).

Meanwhile, minimizing the squared Laplacian energy,

$$
E_{\Delta^{2}}(u)=\frac{1}{2} \int_{\Omega}(\Delta u)^{2} d A,
$$

results in a fourth-order $b i$-Laplace equation with many different combinations of prescribable boundary conditions (values and normal derivatives, values and Laplacians, normal derivatives and Laplacians, etc.). This greater expressive power comes with greater responsibility during modeling.

This greater responsibility manifests itself in the need for carefully choosing boundary conditions for higher order energies, such as the squared Laplacian energy. Indeed, lacking a careful 

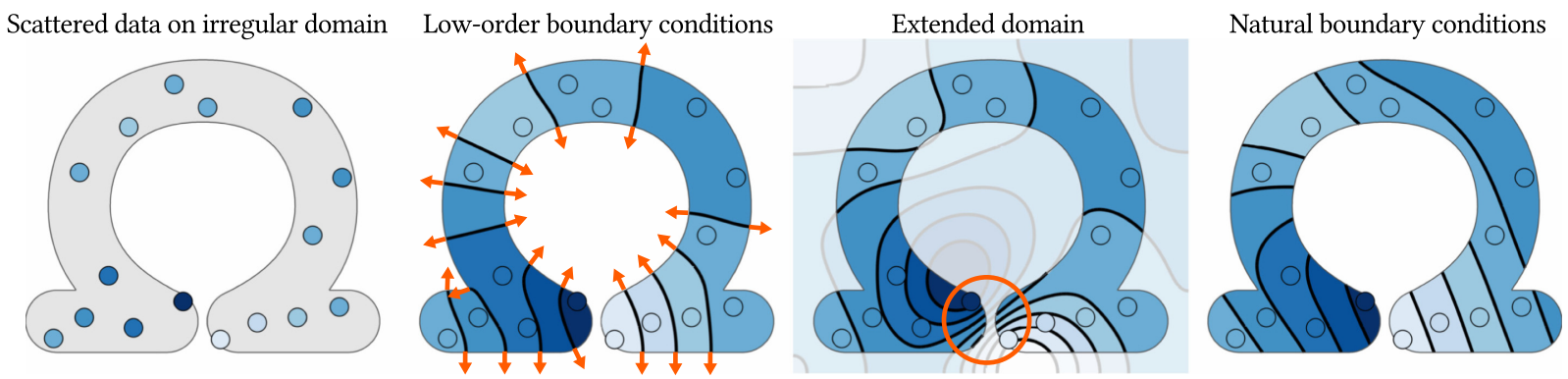

Fig. 2. When interpolating scattered data over a bounded domain, Neumann conditions introduce a noticeable bias at the boundary (orange arrows). Meanwhile, ignoring the boundary by interpolating over ambient space allows bleeding of data onto geodesically distant regions (orange circle).

and systematic treatment of boundary conditions can lead to undesirable and unintuitive artifacts in geometry processing applications. We here refer to boundary conditions that only depend on the function value and its first derivatives, such as Dirichlet and Neumann boundary conditions, as low-order. Boundary conditions involving higher derivatives are referred to as high-order. Consider, for example, the problem of data smoothing. Enforcing Neumann boundary conditions for the squared Laplacian energy leads to noticeable bias along the boundary, which is often unintentional, as illustrated in Figure 1 (middle). Likewise, consider the problem of scattered data interpolation, such as in Figure 2. Here Neumann boundary conditions will cause the interpolation to be strongly influenced by the boundary's shape and isolines will be perpendicular to the boundary (Figure 2, orange arrows). One option would be to simply ignore the given domain's boundary and instead solve over a naive extension into the surrounding space. As a consequence, however, data will bleed across the original domain boundaries (Figure 2, orange circle).

In this article, we offer a study of the effect of boundary conditions for second-order energies derived from the biharmonic equation. In particular, we show limitations of the popular squared Laplacian energy, and we propose an alternative second-order energy: the squared Frobenius norm of the Hessian or simply squared Hessian energy:

$$
E_{\mathbf{H}^{2}}(u)=\frac{1}{2} \int_{\Omega}\left\|\mathbf{H}_{u}\right\|_{F}^{2} d A,
$$

where $\mathbf{H}_{u} \in \mathbb{R}^{2 \times 2}$ is the symmetric matrix of second-order partial derivatives. While this energy appears frequently in the mechanics and image processing literatures, it has not been applied and analyzed as a smoothness energy for geometry processing on irregular domains.

If both Dirichlet and Neumann boundary conditions are enforced simultaneously, then minimizers of $E_{\mathrm{H}^{2}}$ and $E_{\Delta^{2}}$ will be identical. However, these two energies offer dramatically different behavior for "free" or natural boundaries where no explicit boundary conditions are enforced. Natural boundary conditions are those that, among all possible boundary conditions, minimize the given energy.

Unlike the natural boundary conditions of the squared Laplacian energy, those of the squared Hessian energy admit well-behaved solutions without strong bias near the boundary (see Figure 3).
We show the implications of this when modeling smoothness in geometry processing. We derive these boundary conditions and analyze their effects in detail. The advantages of the squared Hessian energy are especially pronounced for irregular domains (e.g., non-convex domains with arbitrarily shaped, non-axisaligned boundaries).

To demonstrate practical results for typical applications of smoothness energies, we discretize the squared Hessian energy with natural boundary conditions, following the same steps that have been used to discretize the squared Laplacian energy previously, using finite differences on $2 \mathrm{D}$ grids and mixed finiteelements on triangle meshes.

Alongside our core contribution of providing a boundaryinsensitive smoothness energy for geometry processing tasks on irregular domains, we also present a novel $L_{1}$-norm minimization for piecewise planar data reconstruction as well as a smooth understanding of previous discrete methods for linear subspace design for real-time shape deformation.

\section{BACKGROUND}

Before discussing related works specifically, we establish concretely what we mean by natural boundary conditions.

\subsection{Definition: Natural Boundary Conditions}

In the calculus of variations, the term natural boundary conditions refers specifically to boundary conditions appearing in the Euler-Lagrange equation of an energy minimization problem that were not imposed beforehand (see p. 26 of Gelfand and Fomin (1963) or p. 34 of Giquinta and Hildebrandt (1996)). This definition is general in that it applies to any energy or partially constrained energy. Just like other types of boundary conditions, it is possible to witness natural boundary conditions on one part of a domain's boundary and explicitly enforce different boundary conditions on another part.

Natural boundary conditions achieve the lowest energy among all possible boundary conditions for a given minimization problem. Consequently, natural boundary conditions heavily depend on the energy being minimized. As we will see, the natural boundary conditions of the squared gradient energy (Dirichlet energy) in Equation (1), the squared Laplacian energy in Equation (2), and Equation (3) are all strikingly different. 

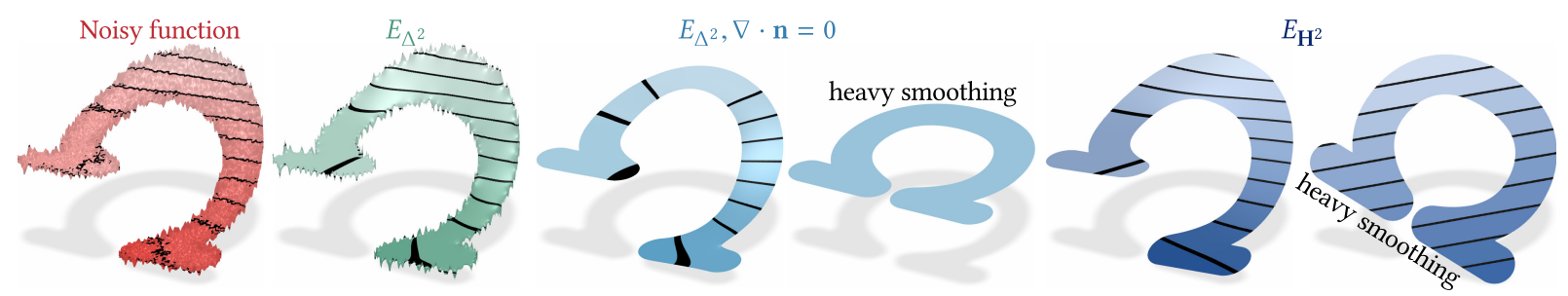

Fig. 3. Smoothing a function over an omega-shaped domain. The high-order natural boundary conditions of $E_{\Delta^{2}}$ maintain noisy boundaries (green). Previous works use low-order boundary conditions, but this biases the solution to follow the boundary even if the input data does not (light blue). Smoothing converges to a constant function. In contrast, the high-order natural boundary conditions of $E_{\mathrm{H}^{2}}$ produce smooth boundary values without bias and converges to a linear function (dark blue).

\subsection{Related Work}

The natural boundary conditions of $E_{\Delta^{2}}$ and $E_{\mathrm{H}^{2}}$ were considered by Courant and Hilbert (1924). Since then, second-derivative energies have been considered in various fields, including data, image, and geometry processing. Earlier work also relates to ours in how the energy, boundary conditions, and domain are chosen, and in their application.

2.2.1 Geometry Processing. High-order smoothness energies are a popular and powerful tool. Discretizations of the squared Laplacian energy have been used for surface fairing (Desbrun et al. 1999), smooth surface displacements (Andrews et al. 2011; Botsch and Kobbelt 2004; Sorkine et al. 2004; Zhou et al. 2005), smooth geodesic distance computation (Lipman et al. 2010), data smoothing (Weinkauf et al. 2010), and regularization during other high-level operations (Cao et al. 2014, 2015; Huang et al. 2006; Jones et al. 2016; Zhou et al. 2010). These and other works square the discrete cotangent Laplacian for triangle meshes (Pinkall and Polthier 1993). This Laplacian matrix L is constructed assuming zero Neumann boundary conditions (i.e., for a boundary edge $i j$ with opposite angle $\alpha_{i j}, L_{i j}=\cot \alpha_{i j}$ ). Squaring this matrix effectively bakes in these conditions. Jacobson et al. (2010) confirm that this construction agrees with a convergent mixed finite element discretization of minimizing $E_{\Delta^{2}}$ subject to zero Neumann boundary conditions $(\nabla u \cdot \mathbf{n}=0)$.

Squaring $\mathrm{L}$ is an intuitive and easy way to handle unconstrained boundaries when employing $E_{\Delta^{2}}$. However, we show that zero Neumann boundary conditions are not natural for $E_{\Delta^{2}}$ according to the mathematical definition in Section 2.1. Perhaps surprisingly, the true natural boundary conditions of $E_{\Delta^{2}}$ are not useful in general (see Figure 3). Meanwhile, the "not natural" zero Neumann conditions are clearly quite useful, as evidenced by the sheer number of geometry processing methods that impose them and achieve great results for their respective applications (Finch et al. 2011; Jacobson et al. 2011, 2012; Kavan et al. 2011; Landreneau and Schaefer 2010; Lipman et al. 2010; Rustamov 2011; Sýkora et al. 2014; Weber et al. 2012; Weinkauf et al. 2010). And yet, we will present evidence that the natural boundary conditions of $E_{\mathbf{H}^{2}}$ can be more useful than either the zero Neumann or natural boundary conditions of $E_{\Delta^{2}}$ in contexts where boundaries should minimally influence the solution.

Natural boundary conditions for energies involving first derivatives are found frequently in geometry processing or related fields such as computer animation (Batty 2010; Bojsen-Hansen and Wojtan 2016). When parameterizing surfaces, natural boundary conditions for the as-conformal-as-possible energy remove the burden of prescribing values for cut-boundaries (Cohen-Steiner and Desbrun 2002; Desbrun et al. 2002; Lévy et al. 2002; Mullen et al. 2008; Springborn et al. 2008). The boundary conditions imposed by Desbrun et al. (2002) have been applied to different energies to which they are no longer natural per Section 2.1, though they nonetheless serve the application (Gingold et al. 2006).

Previous works in geometry processing have considered how to discretize the Hessian of a scalar function defined on a triangulated surface (de Goes et al. 2014; Tosun et al. 2007). These methods do not explore minimization of $E_{\mathrm{H}^{2}}$ or its natural boundary conditions.

Wang et al. (2015) square a modified cotangent Laplacian to design linear subspaces for cartoon animation; they do not explicitly discuss boundary conditions. We will consider the discrete energy in Wang et al. (2015) and its relationship to others built using DEC (Fisher et al. 2007) and non-conforming edge-based elements (Bergou et al. 2006) in Section 4.4. We show that-in contrast to these previous discrete energies-the null space of $E_{\mathrm{H}^{2}}$ contains and only contains affine functions, the necessary and sufficient condition for linear subspace design as set forth by Wang et al. (2015).

In the infinite Euclidean domain, biharmonic radial basis functions (a.k.a., thin-plate splines) minimize $E_{\mathbf{H}^{2}}$ and form a useful basis for various scattered data interpolation problems (Bookstein 1989). Botsch and Kobbelt (2005) applied these globally supported functions to shape deformation and modelling by densely sampling selected surface regions. Free surface boundaries are not noticed any differently from the surface interior as both are embedded in a deformation of the entire Euclidean space (see Figure 4).

2.2.2 Energies and Boundary Conditions in Other Fields. Second-derivative smoothness energies such as $E_{\Delta^{2}}$ and $E_{\mathrm{H}^{2}}$ are found frequently in denoising, restoration, inpainting, image enhancement, and domain reduction applications. Works in image processing that specifically consider $E_{\mathrm{H}^{2}}$ (Didas and Weickert 2004; Lefkimmiatis et al. 2012; Lysaker and Tai 2006; Roth 2009; Terzopoulos 1984) or a broader class of energies including $E_{\mathbf{H}^{2}}$ as a special case (Didas et al. 2009; Lefkimmiatis et al. 2012) impose low-order boundary conditions, with some notable exceptions: Terzopolous $(1984,1988)$ was the first to employ the natural boundary conditions of $E_{\mathbf{H}^{2}}$ in the vision and graphics literature, in the context of surface reconstruction from images. Didas et al. 


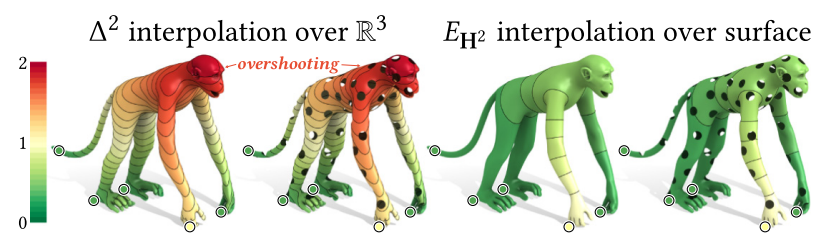

Fig. 4. Scattered data interpolation using biharmonic radial basis functions in $\mathbb{R}^{3}$ ignores the embedded surface and therefore also surface boundaries. In contrast, interpolation by minimizing $E_{\mathrm{H}^{2}}$ on the surface is "shape aware," yet not qualitatively disturbed by open boundaries.

(2004, 2009) invoked the natural conditions for image denoising, whereas Lefkimmiatis et al. (2012) removed boundaries altogether via periodic or reflexive identification. When smoothing over the convex image rectangle, free boundaries have diminished influence on the behavior of the solution. Applications such as inpainting frequently consider non-convex, irregular image subregions, but enforce low-order boundary conditions explicitly to ensure value and derivative continuity (Georgiev 2004).

Other works consider related energies, such as the $L_{1}$-norm of the Hessian (Lysaker et al. 2003; Steidl 2006; Yuan et al. 2009) (a generalization of total variation (Rudin et al. 1992)), the energy $E_{\Delta^{2}}$ (Steidl et al. 2005), or variants thereof (You and Kaveh 2000). We show how the $L_{1}$-norm can analogously apply to smoothing geometric data.

Other authors formulate fourth-order equations directly, not via an energy of second-derivatives. For instance, Liu et al. (2015) explicitly enforce second- and third-order boundary conditions $(\nabla u \cdot \mathbf{n}=\nabla \Delta u \cdot \mathbf{n}=0)$ on a fourth-order equation similar to the biLaplace equation. As we will see in Section 4, this set of boundary conditions is not natural to either $E_{\Delta^{2}}$ or $E_{\mathrm{H}^{2}}$, however, they are frequently imposed on $E_{\Delta^{2}}$ for geometry processing problems on surfaces with boundaries.

$E_{\Delta^{2}}$ and $E_{\mathbf{H}^{2}}$ have been used for the spectral embedding of high dimensional data (Belkin and Niyogi 2004; Donoho and Grimes 2003). A generalization of $E_{\mathbf{H}^{2}}$ was used by Steinke and Hein (2009) for regression, using the energy's natural boundary conditions (therein referred to as implicit).

In physical systems, natural boundary conditions typically have an intuitive explanation: they explain the behavior of free boundaries. In elastic beam theory, natural boundary conditions are higher-order and appear for biharmonic-like equations (see, e.g., Li (2000)). Similar boundary conditions also appear in Kirchhoff plate bending theory (Felippa 2017). We now leverage this physical intuition in one dimension before discussing smoothing in two dimensions and on surfaces.

\section{ONE DIMENSION}

As a didactic exercise, let us derive natural boundary conditions for the one-dimensional squared second derivative energy integrated over the unit line segment:

$$
\min _{u} \frac{1}{2} \int_{0}^{1}\left(u^{\prime \prime}\right)^{2} d x
$$

where $u^{\prime}=d u / d x, u^{\prime \prime}=d^{2} u / d x^{2}$ and so on. Throughout, we appeal to the physical metaphor of a bending bar (see Figure 5).
In practice, we minimize this energy subject to various boundary conditions or additional energy terms. However, applying the calculus of variations to the the energy's raw form will better illustrate natural boundary conditions. In the absence of explicit boundary conditions, then $u$ is a minimizer of this energy if any infinitesimal variation $\varepsilon v$ does not change its energy:

$$
0=\left.\frac{d}{d \varepsilon}\right|_{\varepsilon=0} \frac{1}{2} \int_{0}^{1}\left((u+\varepsilon v)^{\prime \prime}\right)^{2} d x=\int_{0}^{1} u^{\prime \prime} v^{\prime \prime} d x
$$

for all sufficiently smooth functions $v$. In particular, $v$ is not constrained at the boundary. Integration by parts yields

$$
0=\int_{0}^{1} u^{\prime \prime \prime \prime} v d x-\left[u^{\prime \prime \prime} v\right]_{0}^{1}+\left[u^{\prime \prime} v^{\prime}\right]_{0}^{1}
$$

for all $v$. By judiciously testing with $v$ functions, we conclude that $u^{\prime \prime \prime \prime}=0$ on the interior interval $(0,1)$ and $u^{\prime \prime}=u^{\prime \prime \prime}=0$ at the boundary $\{0,1\}$. We began without explicit boundary conditions, so these are the natural boundary conditions.

We observe that functions in the energy's null space satisfy that energy's natural boundary conditions-any linear function $u$ measures zero energy. Correspondingly, we can interpret the natural boundary conditions as "the solution should be linear at the boundary" $\left(u^{\prime \prime}=0\right)$.

We can repeat this process even if we fix some but not all boundary conditions explicitly. For example, if we fix $u(0)=0$ then the remaining natural boundary conditions will be $u^{\prime \prime}(0)=0$ and $u^{\prime \prime}(1)=u^{\prime \prime \prime}(1)=0$, leaving any linear function passing through the origin as a solution. If we also fix $u^{\prime}(0)=0$, then the boundary at $x=0$ is fully constrained and natural boundary conditions only appear at $x=1$, namely $u^{\prime \prime}(1)=u^{\prime \prime \prime}(1)=0$. In this case, the problem has a unique albeit trivial solution: $u=0$, the only linear function passing through the origin with zero slope.

From a mechanics perspective, natural boundary conditions correspond to zero forces and zero moments at the boundary. Figure 5 shows an example of a uniform load applied to a bending bar (i.e., $\left.\min _{u} \int_{0}^{1}\left(u^{\prime \prime}\right)^{2}+u d x\right)$, with various explicit boundary conditions and each problem's resulting set of natural boundary conditions.

In two dimensions, there will be many different ways to construct a smoothness energy out of squared second derivatives. Different energies might even behave the same when boundaries are sufficiently fixed, but imply different natural boundary conditions in the presence of free boundaries.

\section{TWO DIMENSIONS}

Given a flat $2 \mathrm{D}$ domain $\Omega \subset \mathbb{R}^{2}$ with a possibly intricate yet smooth boundary, we consider two intimately related energies that measure second-order variations of a function $u: \Omega \rightarrow \mathbb{R}$ : the squared Laplacian energy $E_{\Delta^{2}}(u)$ of Equation (2) and the squared Hessian energy $E_{\mathrm{H}^{2}}(u)$ of Equation (3).

\subsection{Green's Identities}

We will employ various Green's identities for functions in $\mathbb{R}^{2}$ to move derivatives across inner products by introducing a boundary term. Starting with the classic identity: 


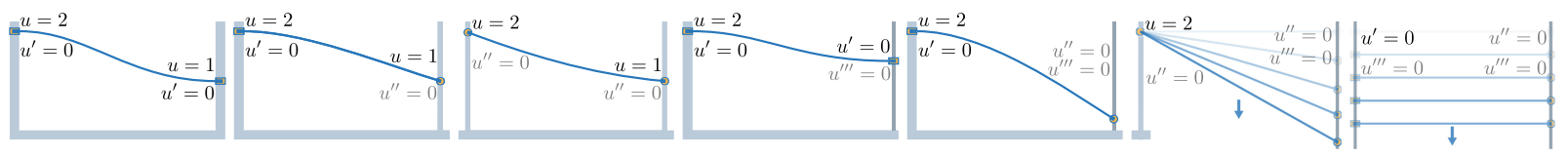

Fig. 5. A bending bar experiences a uniform load subject to various explicit boundary conditions (black, e.g., $u=2$ ); in their absence, natural boundary conditions emerge (gray, e.g., $u^{\prime \prime}=0$ ). This physical problem has a unique solution when at least two low-order boundary conditions are imposed; otherwise, the problem becomes underconstrained and the solution tends toward infinite values. Calculus of variation shows that a uniform load does not change the natural boundary conditions.

$$
\int_{\Omega}(\nabla u \cdot \nabla v+u \Delta v) d A=\oint_{\partial \Omega} u \nabla v \cdot \mathbf{n} d s,
$$

replacing $u$ in Equation (7) with $\Delta u$ :

$$
\int_{\Omega}(\nabla \Delta u \cdot \nabla v+\Delta u \Delta v) d A=\oint_{\partial \Omega} \Delta u \nabla v \cdot \mathbf{n} d s,
$$

replacing $v$ in Equation (7) with $\Delta v$ :

$$
\int_{\Omega}\left(\nabla u \cdot \nabla \Delta v+u \Delta^{2} v\right) d A=\oint_{\partial \Omega} u \nabla \Delta v \cdot \mathbf{n} d s,
$$

replacing $u$ and $v$ in Equation (7) with $\nabla u^{\top}$ and $\nabla v^{\top}$ :

$$
\int_{\Omega}\left(\mathbf{H}_{u}: \mathbf{H}_{v}+\nabla u \cdot \nabla \Delta v\right) d A=\oint_{\partial \Omega} \nabla u^{\top} \mathbf{H}_{v} \mathbf{n} d s,
$$

replacing $u$ and $\nabla v$ in Equation (7) with a vector $\mathbf{u}$ and matrix $\mathbf{V}$ :

$$
\int_{\Omega}(\nabla \mathbf{u}: \mathbf{V}+\mathbf{u} \cdot(\nabla \cdot \mathbf{V})) d A=\oint_{\partial \Omega} \mathbf{u}^{\top} \mathbf{V n} d s,
$$

where $\mathrm{X}: \mathrm{Y}:=\operatorname{tr}\left(\mathrm{X}^{T} \mathrm{Y}\right)$ computes the Frobenius inner product, which generates the Frobenius norm $\mathrm{X}: \mathrm{X}=\|\mathrm{X}\|_{F}^{2}$, and $\nabla \cdot \mathrm{X}$ computes the matrix divergence of $\mathrm{X}$.

\subsection{Equivalence up to Boundary Conditions}

We first show that-regardless of boundary conditionsminimizers of both $E_{\Delta^{2}}$ and $E_{\mathbf{H}^{2}}$ satisfy the biharmonic equation $\left(\Delta^{2} u=0\right)$ in the interior of the domain. To avoid redundant derivations, we introduce a parameter $\alpha$ (cf. Courant and Hilbert (1924) and Terzopoulos (1984)) so that setting $\alpha=1$ gives $E_{\mathbf{H}^{2}}$ and $\alpha=0$ gives $E_{\Delta^{2}}$ :

$$
\frac{1}{2} \int_{\Omega}\left((1-\alpha)(\Delta u)^{2}+\alpha\left\|\mathbf{H}_{u}\right\|_{F}^{2}\right) d A .
$$

As in Section 3, $u$ is a minimizer if adding any infinitesimal variation $\varepsilon v$ will not change its energy. Thus,

$$
0=\int_{\Omega}\left((1-\alpha) \Delta u \Delta v+\alpha \mathbf{H}_{u}: \mathbf{H}_{v}\right) d A
$$

for all $v$. Applying Equations (8) and (10), this becomes

$$
\begin{aligned}
0= & \int_{\Omega}(-(1-\alpha) \nabla \Delta u \cdot \nabla v-\alpha \nabla v \cdot \nabla \Delta u) d A \\
& +\oint_{\partial \Omega}\left((1-\alpha) \Delta u \nabla v \cdot \mathbf{n}+\alpha \nabla v^{\top} \mathbf{H}_{u} \mathbf{n}\right) d s
\end{aligned}
$$

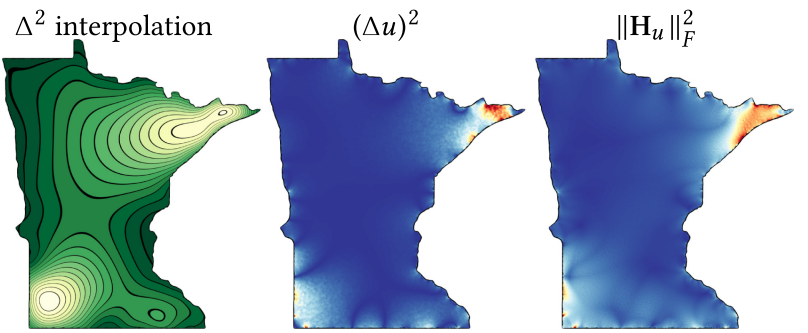

Fig. 6. Subject to fixed boundary values and normal derivatives, the same biharmonic interpolation minimizes both $E_{\Delta^{2}}$ and $E_{\mathrm{H}^{2}}$, despite each energy measuring a different local quantities.

for all $v$. Then applying Equation (9), we arrive at

$$
\begin{aligned}
0= & \int_{\Omega} v \Delta^{2} u d A \\
& +\oint_{\partial \Omega}\left(-v \nabla \Delta u \cdot \mathbf{n}+(1-\alpha) \Delta u \nabla v \cdot \mathbf{n}+\alpha \nabla v^{\top} \mathbf{H}_{u} \mathbf{n}\right) d s
\end{aligned}
$$

for all $v$. Because this equality must hold for any choice of $v, u$ must be biharmonic in the interior, regardless of $\alpha$ :

$$
\Delta^{2} u=0 \text { on } \Omega \text {. }
$$

Biharmonic functions are uniquely determined by boundary values and normal derivatives (Evans 1998, pp. 345), therefore minimizers of $E_{\Delta^{2}}$ and $E_{\mathbf{H}^{2}}$ on $\mathbb{R}^{2}$ will be identical when explicit low-order boundary conditions are prescribed, i.e., fixing Dirichlet $(u=f)$ as well as Neumann $(\nabla u \cdot \mathbf{n}=g)$ conditions. Indeed, enforcing such boundary conditions during calculus of variations requires considering variations such that $v=\nabla v \cdot \mathbf{n}=0$ on the boundary. This immediately implies that $\nabla v=0$ on the boundary and, correspondingly, that the entire boundary integral in Equation (15) vanishes. In conclusion, when modeling smoothness for boundary-value interpolation problems, these energies are interchangeable despite measuring different local quantities (see Figure 6).

\subsection{Natural Boundary Conditions}

In the absence of explicit boundary conditions, the variations $v$ in Equation (15) include all sufficiently smooth functions. We choose from them specific variations to expose the natural boundary conditions of the parameterized family of energies in Equation (12). First, consider all $v$ that vanish on the boundary but whose gradient exists only in the normal direction $\left(v=0\right.$ and $\nabla v=\left.g \mathbf{n}\right|_{\partial \Omega}$, for 


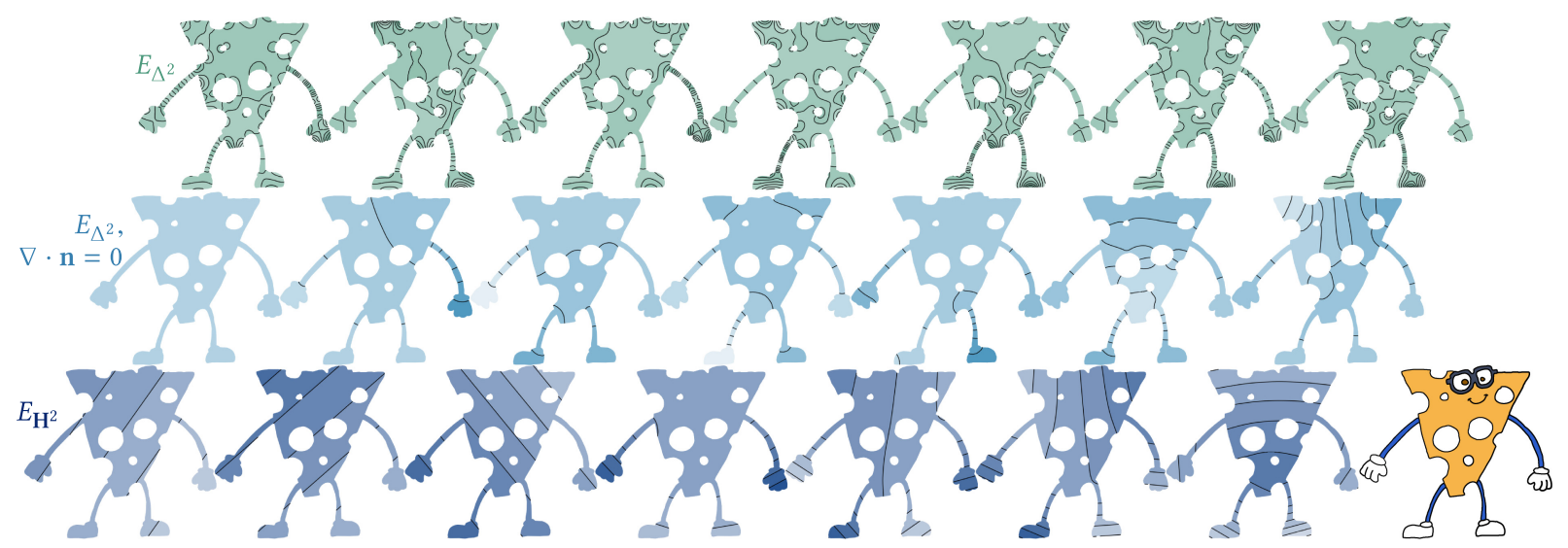

Fig. 7. Ten lowest frequency modes of $E_{\Delta^{2}}$ with natural boundary conditions on a 2D Cheeseman include random harmonic functions with high oscillation near the boundary (top). Adding zero Neumann boundary conditions to $E_{\Delta^{2}}$ causes modes to reproduce familiar Laplacian eigenfunctions (middle). In contrast, modes of $E_{\mathrm{H}^{2}}$ with natural boundary conditions include affine functions and other low frequency functions without constrained normal derivatives (bottom).

some arbitrary function $g$ ). Second-order natural boundary conditions must hold:

$$
(1-\alpha) \Delta u+\alpha \mathbf{n}^{\top} \mathbf{H}_{u} \mathbf{n}=0 \text { on } \partial \Omega .
$$

Additionally, considering all $v$ with zero normal derivative without restricting the value of $v$ along the boundary $\left(\nabla v \cdot \mathbf{n}=\left.0\right|_{\partial \Omega}\right)$, we witness third-order boundary conditions must hold:

$$
\nabla \Delta u \cdot \mathbf{n}+\alpha \nabla\left(\mathbf{t}^{\top} \mathbf{H}_{u} \mathbf{n}\right) \cdot \mathbf{t}=0 \text { on } \partial \Omega .
$$

Both conditions depend on $\alpha$, and therefore the natural boundary conditions for the squared Laplacian and squared Hessian energies indeed differ (ref. (Courant and Hilbert 1924)). We now build an intuition for these conditions for canonical choices of $\alpha$ and contrast their behavior when modeling smoothness on domains with free boundaries.

\subsection{Contrasting Natural Boundary Behavior}

For the squared Laplacian energy $E_{\Delta^{2}}(\alpha=0)$, unconstrained minimizers of $E_{\Delta^{2}}(\alpha=0)$ will be biharmonic on the interior $\left(\Delta^{2} u=0\right)$ and satisfy natural boundary conditions forcing the solution to be harmonic along the boundary $(\Delta u=0)$ and continue to be harmonic across the boundary $(\nabla \Delta u \cdot \mathbf{n}=0)$. Consistent with our understanding from Section 2.1, these boundary conditions agree with the energy's null space: any harmonic function $u$ measures zero energy $E_{\Delta^{2}}(u)=0$.

For the squared Hessian energy $E_{\mathrm{H}^{2}}(\alpha=1)$, unconstrained minimizers are also biharmonic on the interior $\left(\Delta^{2} u=0\right)$, but satisfy different natural boundary conditions, forcing the function to be linear in the normal direction $\left(\mathbf{n}^{\top} \mathbf{H}_{u} \mathbf{n}=0\right)$ and after that continue with low variation along the boundary $\nabla \Delta u \cdot \mathbf{n}+\nabla\left(\mathbf{t}^{\mathrm{T}} \mathbf{H}_{u} \mathbf{n}\right) \cdot \mathbf{t}=0$. Again, this agrees with the null space: all linear functions measure zero energy.

The null space of $E_{\Delta^{2}}$ is infinite dimensional: for any boundary values, there exists a harmonic function interpolating them. This renders the $E_{\Delta^{2}}$ with natural boundary conditions rather useless as a smoothness energy. For data smoothing, noisy data along the boundary will simply remain, since there exists an interpolating harmonic function that can be added without affecting the energy (see Figure 3, left).

In contrast, the linear functions in the null space of $E_{\mathbf{H}^{2}}$ are finite dimensional and intuitive. For data smoothing, this null space models "in the absence of any other information, fit a linear function" (see Figure 3, right).

Low-frequency eigenfunctions of smoothness energies are a standard way to construct a smooth low-frequency function space in geometry processing. Typically, previous works use the loworder Dirichlet energy in Equation (1) with its natural boundary conditions $(\nabla u \cdot \mathbf{n}=0)$ (Hildebrandt et al. 2011; Zhang et al. 2007). Such modal analysis on the higher-order $E_{\Delta^{2}}$ with its natural boundary conditions is not practical because low-frequency modes are polluted with arbitrary harmonic functions (see Figure 7, top). Explicitly enforcing zero Neumann boundary conditions $\nabla u \cdot \mathbf{n}=0$ on $E_{\Delta^{2}}$ alleviates this but simply results in the same spectrum as $E_{\nabla^{2}}$ (up to squared eigenvalues, Lipman et al. (2010)). In contrast, the low frequency modes of $E_{\mathbf{H}^{2}}$ provide a new, smooth, and well-behaved basis (see Figure 7).

4.4.1 Mixing with Explicit Boundary Conditions. We examine how explicitly enforcing certain boundary conditions changes the remaining natural boundary conditions. For example, to explicitly enforce values of $u$ along the boundary (Dirichlet conditions), we must assume that our test function $v$ vanishes on the boundary $\left(v=\left.0\right|_{\partial \Omega}\right.$ in Equations (13)-(15)). Only second-order natural boundary conditions remain. For $E_{\Delta^{2}}$, the additional natural boundary conditions are $\Delta u=0$. For $E_{\mathbf{H}^{2}}$, they are $\mathbf{n}^{\top} \mathbf{H}_{u} \mathbf{n}=0$.

As discussed in Section 2, many previous works in geometry processing minimize the squared Laplacian energy $E_{\Delta^{2}}$ subject to zero Neumann conditions $\left(\left.\nabla u \cdot \mathbf{n}\right|_{\partial \Omega}=0\right)$. We obtain that the only additional natural boundary conditions are third-order: $\nabla \Delta u \cdot \mathbf{n}=0$.

For data smoothing, zero Neumann boundary conditions encourage functions to be flat near the boundary. The null space correspondingly shrinks to contain only constant functions. If the smoothness energy dominates over the data, then a best fit 

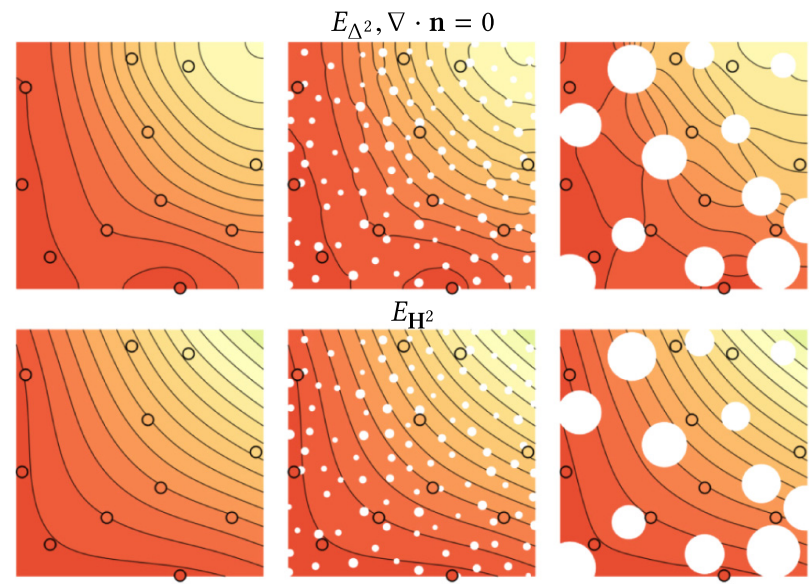

Fig. 8. Interpolating scattered samples of a quadratic function over a square domain without holes (left) and with holes (center and right). When minimizing $E_{\Delta^{2}}$ with Neumann boundary conditions, the presence and shape of boundaries effects the solution. With $E_{\mathrm{H}^{2}}$, boundaries are less noticeable.

constant function is found (see Figure 3, center). For arbitrary domains, the zero Neumann boundary conditions create a strong boundary sensitivity (see Figure 8).

\subsection{Relationship to Previous Discrete Energies}

The null space of the squared Hessian energy $E_{\mathrm{H}^{2}}$ contains and only contains linear functions. In the context of real-time shape deformation, Wang et al. (2015) specify that this is precisely the condition needed of a smoothness energy for linear subspace design. With this property as a goal, Wang et al. (2015) and other previous works have designed discrete energies by modifying the discrete cotangent Laplace operator, $\mathrm{L} \in \mathbb{R}^{n \times n}$, for a triangle mesh with $n$ vertices and $k$ edges. The unmodified discrete Laplace operator $\mathbf{L}$ can be derived via a finite-element discretization of the squared gradient energy in Equation (1), and the discrete energy preserves the smooth structure of having only constant functions in its null space. In contrast, the following two discrete modifications of $\mathbf{L}$ do not have known smooth analogs, and as such are more susceptible to errors and more challenging to analyze (Wang et al. 2017).

4.5.1 Discrete Exterior Calculus. Fisher et al. (2007) construct a discrete energy for tangent vector field design using discrete exterior calculus (DEC) on triangle meshes. Their energy measures the sum of all squared discrete divergences of a given vector field v via

$$
\mathbf{v}^{\top} \star_{1} d_{0} \star_{0}^{-1} \underbrace{d_{0}^{\top} \star_{1}}_{\mathbf{F}} \mathbf{v},
$$

where $\star_{0}, \star_{1}$ and $d_{0}$ are the discrete Hodge star operators for zeroand one-forms and the discrete differential matrix for one-forms, respectively, following the adjustments when building $\mathbf{F}$ for free boundaries as described in Fisher et al. (2007). If we replace the unknown vector field $\mathbf{v}$ with the differential of an unknown scalar field $d_{0} \mathbf{u}$, we can build a discrete smoothness energy of a scalar

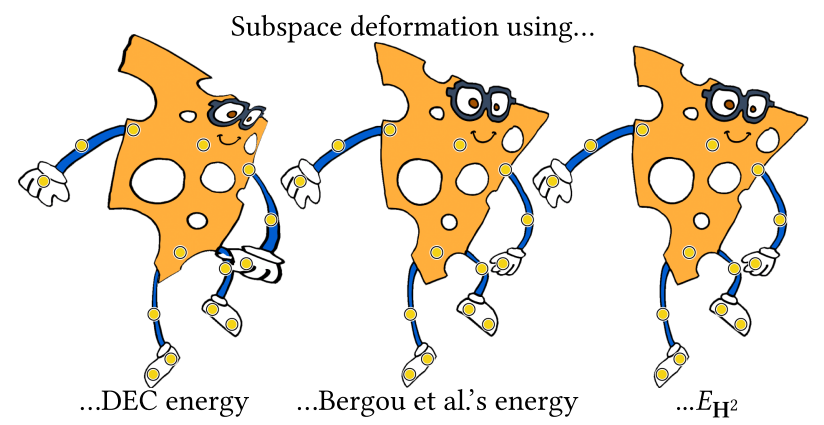

Fig. 9. The smoothness energy built using discrete exterior calculus in Equation (20) is not suitable for linear subspace design. In contrast, deformations using Equation (21) or $E_{\mathrm{H}^{2}}$ are similarly appropriate.

field:

$$
\mathbf{u} d_{0}^{\top} \mathbf{F}^{\top} \underbrace{\star_{0}^{-1}}_{\mathbf{M}^{-1}} \underbrace{\mathbf{F} d_{0}}_{\mathbf{K}=\mathbf{L}+\mathbf{N}} \mathbf{u},
$$

where the matrix $\mathbf{M} \in \mathbb{R}^{n \times n}$ is the (lumped) per-vertex mass matrix, and the non-symmetric matrix $\mathrm{K} \in \mathbb{R}^{n \times n}$ matches the description of the operator in Wang et al. (2015), constructed by adding the normal derivative operator $\mathrm{N} \in \mathbb{R}^{n \times n}$ to the standard cotangent Laplacian $\mathbf{L}$. This same matrix $\mathbf{K}$ has been previously shown to contain at least linear functions in its right null space (Crane 2009).

Unfortunately, for some meshes this matrix-and by extension the energy it defines-contains other non-linear functions in its null space (see inset). This does not appear to be a simple matter of mesh resolution or numerics. From the point of view of linear subspace design (see Figure 9), this null space is too big.

Following the DEC construction, tak-

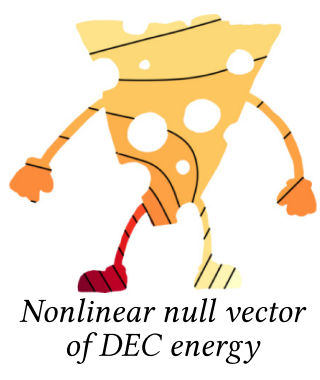
ing the divergence of the gradient should correspond to the smooth Laplace operator $(\Delta=\nabla \cdot \nabla)$. If the discrete energy in Equation (20) were structure preserving, then we would expect it to produce discrete natural boundary conditions for the squared Laplacian energy $E_{\Delta^{2}}$ and match its null space of all harmonic functions (see Figure 7). However, eigen analysis of this energy for typical meshes produces a small number $(\geq 3)$ of null modes. From a structure preservation standpoint, this null space is too small.

4.5.2 Non-Conforming Finite Elements. Wang et al. (2015) use an alternative construction to create their results (Wang et al. 2017). Normal derivatives are added to the edge-based Laplacian $\mathrm{L}_{\mathrm{cr}} \in \mathbb{R}^{k \times k}$ resulting from the Crouzeix-Raviart non-conforming finite-element discretization of the squared gradient energy in Equation (1):

$$
\mathbf{u}^{\mathrm{T}} \mathbf{E}^{\mathrm{T}} \mathbf{K}_{\mathrm{cr}}^{\mathrm{T}} \mathbf{M}_{\mathrm{cr}}^{-1} \underbrace{\mathbf{K}_{\mathrm{cr}}}_{\mathbf{L}_{\mathrm{cr}}+\mathbf{N}_{\mathrm{cr}}} \mathbf{E u},
$$

where $\mathbf{E} \in \mathbb{R}^{k \times n}$ averages vertex values onto incident edges, $\mathbf{M}_{\mathrm{cr}} \in$ $\mathbb{R}^{k \times k}$ is the Crouzeix-Raviart mass matrix, and $\mathrm{N}_{\mathrm{cr}} \in \mathbb{R}^{k \times k}$ computes normal derivatives at boundary edges. 


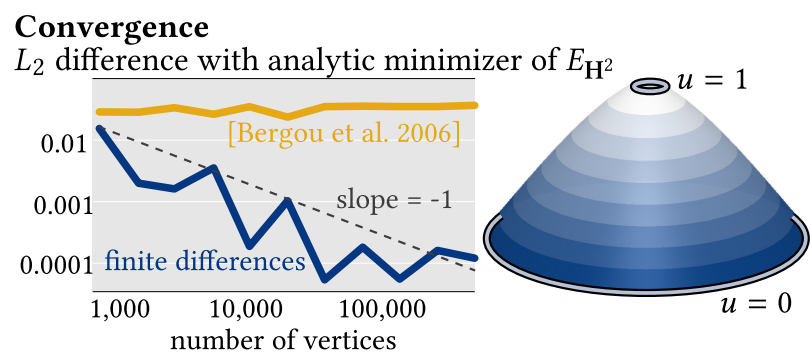

Fig. 10. On a 2D annulus, fixing inner boundary values to one and outer boundary values to zero, the analytic minimizer of $E_{\mathrm{H}^{2}}$ is a radially symmetric function with natural third-order boundary conditions (see Section 4.4.1). While a finite difference discretization of $E_{\mathrm{H}^{2}}$ converges under refinement, minimizing the discrete energy in Bergou et al. (2006) does not.

This energy corresponds to one of two discrete energies proposed by Bergou et al. (2006) to model plate bending under the assumption of isometric deformation as the squared Laplacian $\left(E_{\Delta^{2}}\right)$ of the displacement coordinate functions. Bergou et al. do not discuss boundary conditions and the discrete boundary conditions of their two proposed discrete energies differ (the other corresponding to $E_{\triangle^{2}}$ subject to zero-Neumann boundary conditions).

Perhaps surprisingly, the discrete energy in Equation (21) behaves very similarly to the squared Hessian energy $E_{\mathbf{H}^{2}}$, despite its motivation by both Bergou et al. (2006) and Wang et al. (2015) as a discrete analog to $E_{\Delta^{2}}$. In contrast to the DEC energy in Equation (20), empirical tests indicate that the null space of the Bergou et al. energy contains and only contains linear functions. Resulting subspace deformations are visually indistinguishable from those using $E_{\mathrm{H}^{2}}$ (see Figure 9).

Unfortunately, while the discrete energy in Equation (21) clearly does not behave like a discretization of $E_{\Delta^{2}}$ with natural boundary conditions, it also does not converge to smooth minimizers of $E_{\mathrm{H}^{2}}$ with its natural boundary conditions (see Figure 10). It remains unclear-and outside our scope-to show whether this discrete energy converges to yet some other continuous energy.

\section{DISCRETIZATION}

For our experiments, we discretize the energies $E_{\Delta^{2}}$ and $E_{\mathrm{H}^{2}}$ using either the finite difference method on regular $2 \mathrm{D}$ grids restricted to a bounded subregion $\Omega \subset \mathbb{R}^{2}$ or the mixed finite element method on irregular triangle meshes.

\subsection{Finite Differences}

For finite differences on a 2D regular grid, we use standard central differences to compute each entry of the Hessian at all interior nodes (Fornberg 1988) (see Appendix A).

Our discretization on grids is in agreement with those used in image processing on rectangular domains (Didas et al. 2009). As expected, this standard finite difference discretization converges under refinement (see Figure 10). No special treatment is required in the case of non-convex, irregular domains beyond ensuring that all interior nodes have neighboring nodes in all eight directions.

\subsection{Mixed Finite Elements}

The common discretization of the squared Laplacian energy with zero Neumann boundary conditions on a mesh with $n$ vertices and $m$ faces is constructed by squaring the cotangent Laplacian $\mathrm{L} \in \mathbb{R}^{n \times n}$

$$
E_{\Delta^{2}}(u) \text { subject to } \nabla u \cdot \mathbf{n}=\left.0\right|_{\partial \Omega} \approx \mathbf{u}^{\top} \mathbf{L}^{\top} \mathbf{M}^{-1} \mathbf{L u},
$$

where $\mathbf{M} \in \mathbb{R}^{n \times n}$ is a (often lumped) mass matrix. Jacobson et al. (2010) demonstrated that this discrete energy can be derived by applying the mixed finite element method to the continuous energy minimization problem.

Following their steps, we can similarly discretize the natural boundary conditions ${ }^{1}$ of the squared Laplacian energy:

$$
E_{\Delta^{2}}(u) \approx \mathbf{u}^{\mathrm{T}} \mathbf{L}(i, a)^{\mathrm{T}} \mathbf{M}(i, i)^{-1} \mathbf{L}(i, a) \mathbf{u},
$$

where $\mathbf{X}(j, k)$ indicates slicing rows and columns corresponding to vertex lists $j$ and $k$, respectively, and, specifically, $i$ and $a$ are the lists of interior vertices and all vertices, respectively. It is clear from this construction that any discrete harmonic function $(\mathbf{L}(i, a) \mathbf{u}=$ 0 ) will measure zero energy regardless of boundary values. This is correct from the point of view of structure preservation, and validates the arbitrary boundary behavior observed when using these natural boundary conditions for smoothing (see Figure 3).

We can follow similar steps to use the mixed finite element method to discretize the squared Hessian energy. Beginning in the smooth setting, we introduce an auxiliary matrix-valued variable $\mathrm{V}$ constrained to be equal to the Hessian of the unknown function on the interior of the domain via a matrix-valued Lagrange multiplier function $\Lambda$, which is free on the interior and clamped to zero on the boundary:

$$
\underset{u, \mathrm{~V}, \Lambda}{\operatorname{saddle}} \int_{\Omega}\left(\frac{1}{2} \mathbf{V}: \mathbf{V}+\Lambda:\left(\mathbf{H}_{u}-\mathbf{V}\right)\right) d A, \quad \text { s.t. } \Lambda=\left.0\right|_{\partial \Omega} .
$$

Applying the Green's identity from Equation (11) to the $\Lambda: \mathbf{H}_{u}$ term, we move a derivative from $u$ to $\Lambda$ :

$$
\begin{gathered}
\underset{u, \mathrm{~V}, \Lambda}{\text { saddle }} \int_{\Omega}\left(\frac{1}{2} \mathrm{~V}: \mathrm{V}-(\nabla \cdot \Lambda) \cdot \nabla u-\Lambda: \mathrm{V}\right) d A \\
+\oint_{\partial \Omega} n^{\top} \Lambda \nabla u d s .
\end{gathered}
$$

Applying a functional variation to $\mathrm{V}$, we immediately see that the solution must be obtained when $\mathrm{V}=\left.\Lambda\right|_{\Omega}$, so we can substitute $\Lambda$ out:

$$
\underset{u, \mathrm{~V}}{\operatorname{saddle}} \int_{\Omega}\left(-\frac{1}{2} \mathbf{V}: \mathbf{V}-(\nabla \cdot \mathrm{V}) \cdot \nabla u\right) d A .
$$

This saddle problem involves only first derivatives. We may use standard piecewise-linear elements for $u$ and each of the four coordinate functions of $\mathbf{V}$ (with $\mathbf{V}=\left.0\right|_{\partial \Omega}$ because $\Lambda=\left.0\right|_{\partial \Omega}$ ). Factoring out the degrees of freedom corresponding to $\mathrm{V}$, we have a

\footnotetext{
1Jacobson et al. (2010) mention "natural" boundary conditions for $E_{\Delta^{2}}$ but do not explicitly derive or discretize them. The free boundaries in their results appear to enforce zero Neumann boundary conditions.
} 


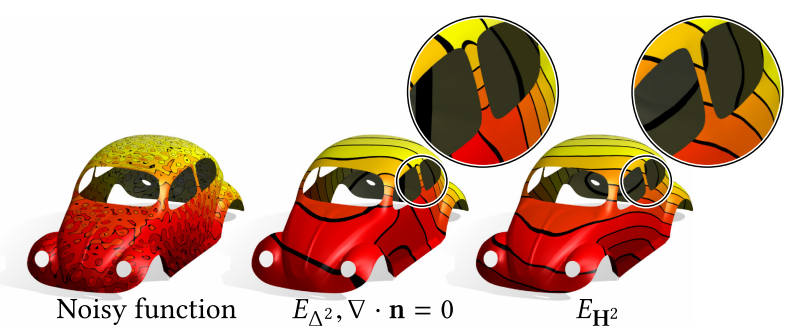

Fig. 11. Low-order boundary conditions are the de facto standard for smoothing in geometry processing (middle), but they introduce bias at the boundary (inset). A naïve extension of our discrete Hessian energy to 3D seems to alleviate this.

discretization of the squared Hessian energy with natural boundary conditions:

$$
E_{\mathbf{H}^{2}}(u) \approx \mathbf{u}^{\top} \mathrm{G}^{\top} \mathrm{AD} \tilde{M}^{-1} \mathrm{D}^{\top} \mathrm{AGu},
$$

where G,A,D, and $\tilde{\mathbf{M}}$ are the discrete gradient operator, diagonal matrix of triangle areas, discrete matrix divergence operator and discrete mass matrix (see Appendix B).

5.2.1 Alternative Discretizations. Many other mixed finiteelement methods have been applied to the biharmonic equation and associated energies in the past (Lamichhane 2011; Scholz 1978). The Hellan-Herrmann-Johnson mixed formulation (see, e.g., Comodi (1989)) has seen recent renewed interest when applied in conjunction with the discontinuous Galerkin method (Braess et al. 2017; Hoppe et al. 2016).

Conforming high-order elements are another option. For example, the Argyris element (Braess 2002) would directly allow secondorder differentiation in $\mathbb{R}^{2}$. Besides introducing a large number of degrees of freedom, it is dependent on the 2D Cartesian coordinate system, making it difficult to extend to arbitrary surfaces.

Higher-order smoothing energies have also been discretized without the finite element method. For example, in machine learning, Kim et al. (2009) discretize the Hessian energy using a nonlinear fitting approach.

\subsection{Triangle Meshes in 3D}

So far, we have only considered flat domains $\Omega \subset \mathbb{R}^{2}$. First experiments suggest that curved surfaces, and especially those with boundaries, might benefit analogously from our analysis of smoothness energies (see Figure 11). We can trivially extend our mixed finite-element discretization of $E_{\mathrm{H}^{2}}$ in Section 5.2 to triangle meshes immersed in $\mathbb{R}^{2}$ by extending the gradient and matrix divergence operators in Equation (26) to compute 3D rather than $2 \mathrm{D}$ vectors. This amounts to temporarily treating the Hessian as a $3 \times 3$ matrix. This extension is inspired by the construction of the discrete Laplacian for surfaces built by trivially extending the gradient operator to compute per-face $3 \mathrm{D}$ vectors while maintaining the property that: $\mathrm{L}=\mathrm{G}^{\top} \mathrm{AG}$. Our discretization of the Hessian and squared Hessian matrices is available open source as part of LIBIGL (Jacobson 2017).

5.3.1 Future Work: Accounting for Curvature. On curved surfaces, the formulation of the Hessian energy is far more intricate.
One striking difference to the planar case arises from the fact that the Hessian energy on smooth surfaces without boundary is in general no longer equal to the Laplacian energy. Indeed, integration by parts similar to Equation (10) leads to the so-called Bochner-Laplacian, which differs from the usual (Hodge-) Laplacian by a term involving Gaussian curvature. Also the naïve extension of our mixed finite element discretization of $E_{\mathrm{H}^{2}}$ to 3D leads to a different

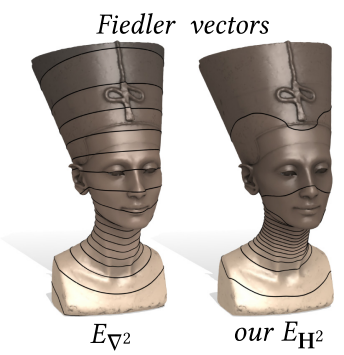
energy than the Laplacian energy (see inset). However, we do not claim this extension to be a proper discrete version of the smooth Hessian energy.

Another complication arises from the fact that even a weak formulation of the Hessian similar to Equation (24) involves covariant differentiation in the smooth setting. One future direction might be to use recent discretizations of the covariant derivative (e.g., Azencot et al. (2015) and Liu et al. (2016)) to derive a discrete Hessian energy while maintaining analogous natural boundary conditions. The original Green's identity in Equation (7) does not introduce curvature terms, allowing discretization of the squared Laplacian Energy using mixed FEM as used for thin shells (Bergou et al. 2006; Garg et al. 2007).

Regardless, first experiments suggest that our naïve extension to 3D might enable our main results concerning free boundaries to operate on surface meshes. We leave an analysis of our naïve extension to 3D for future work.

\section{4 $L_{1}$ Minimization}

Instead of minimizing the $L_{2}$-norm of the Hessian, the squared Hessian energy, we can also minimize its $L_{1}$-norm:

$$
\int_{\Omega}\left|\mathbf{H}_{u}\right|_{F} d A .
$$

This $L_{1}$-type energy exhibits different properties from the ones of the squared Hessian energy (see Section 6).

A detailed description the dizcretization can be found in Appendix C. This is a simple discretization; other discretizations of the $L^{1}$ norm are discussed in works such as Bronstein et al. (2016).

\section{EXPERIMENTS AND APPLICATIONS}

Smoothness energies are a fundamental ingredient in geometry processing algorithms. We tour applications using the Hessian to define smoothness. We solve the resulting sparse linear systems using MATLAB and quadratic programs using MoseK (Andersen and Andersen 2000). We verified that both our discretizations (see Section 5) converge toward the analytic minimizer of $E_{\mathrm{H}^{2}}$ on an annular domain with radially symmetric fixed value and third-order natural boundary conditions (see, e.g., Figure 10). The computational complexity of minimizing $E_{\mathrm{H}^{2}}$ is equivalent to minimizing $E_{\Delta^{2}}$ : in both cases the number of non-zeros per-row is less than or equal to the size of the corresponding vertex's two-neighborhood.

The simplest demonstration of these energies is to reconstruct a smooth function while interpolating values at specific points. For 


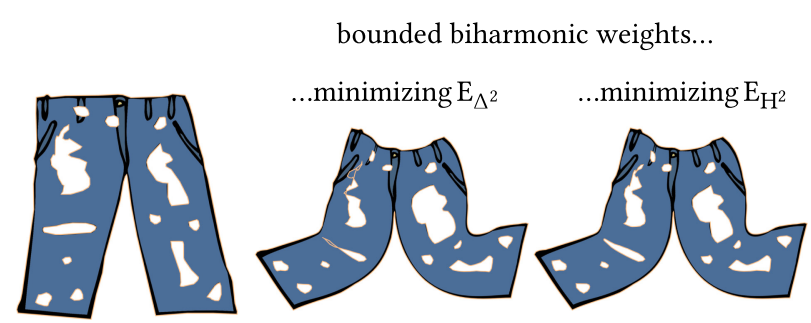

Fig. 12. Both weights are constrained to agree on the outer boundary. On the interior holes, $E_{\mathbf{H}^{2}}$ causes less distortion than $E_{\Delta^{2}}$.

flat domains with interpolated values and normal derivatives along the boundary, the reconstructions minimizing $E_{\Delta^{2}}$ and $E_{\mathbf{H}^{2}}$ will agree (see Figure 6). If the boundary is left unconstrained or partially constrained then natural boundary conditions will appear for each respective energy (see Figure 2). In all examples, differences are best identified by observing how isolines meet with the boundary: zero Neumann boundary conditions cause the isolines to be perpendicular to the boundary, while high-order natural boundary conditions do not force this behavior (see Figure 8).

Linear subspace design for cartoon deformation is a special case of scattered data interpolation. Instead of interpolating colors or temperatures, displacements are interpolated over a 2D cartoon or surface (Jacobson et al. 2014). Minimizing $E_{\mathbf{H}^{2}}$ with its natural boundary conditions and Kronecker delta values at specified point handles, we can define a linear basis for smooth deformation interpolation displacements at these points. Like previous approaches (Wang et al. 2015), the minimizing functions of $E_{\mathrm{H}^{2}}$ are linearly precise and therefore form a form of cage-free generalized barycentric coordinates ("Hessian coordinates"). In contrast to previous work, like the coordinates of Wang et al. (2015), Hessian coordinates are defined by a smooth energy where it is easy to show that all and only linear functions exist in its null space (see Figure 7). Figure 9 shows that the deformation behavior is similar that of Wang et al. (2015).

Similarly, automatic methods for computing linear blend skinning weights have employed smoothness energies (Baran and Popović 2007). Classic bounded biharmonic weights minimize $E_{\Delta^{2}}$ subject to zero Neumann boundary conditions (Jacobson et al. 2011) and bound constraints. Restricting $E_{H^{2}}$ to the same value as classic bounded biharmonic weights on the outside of the shape, but not on interior holes leads to weights that preserve the characteristic shape of holes without much distortion. As can be seen in Figure 12, bounded biharmonic weights based on $E_{\Delta^{2}}$ distort the interior holes of the pants significantly under deformation, while $E_{H^{2}}$ alleviates the effect.

We now turn our attention to another common use for a smoothness energy: dense data denoising or fairing. To do so, we can optimize a function $u$ that minimizes the weighted sum of a data term $\left(L_{2}\right.$-norm of difference between $u$ and a noisy input function $f$ ) and the $L_{2}$ smoothness energy (e.g., $E_{\Delta^{2}}$ or $E_{\mathbf{H}^{2}}$ ). When using $E_{\Delta^{2}}$ for data smoothing on domains with free boundaries, previous methods (e.g., Weinkauf et al. (2010)) enforce low-order boundary conditions to ensure that noisy boundary values are not simply interpolated (see Figure 3).

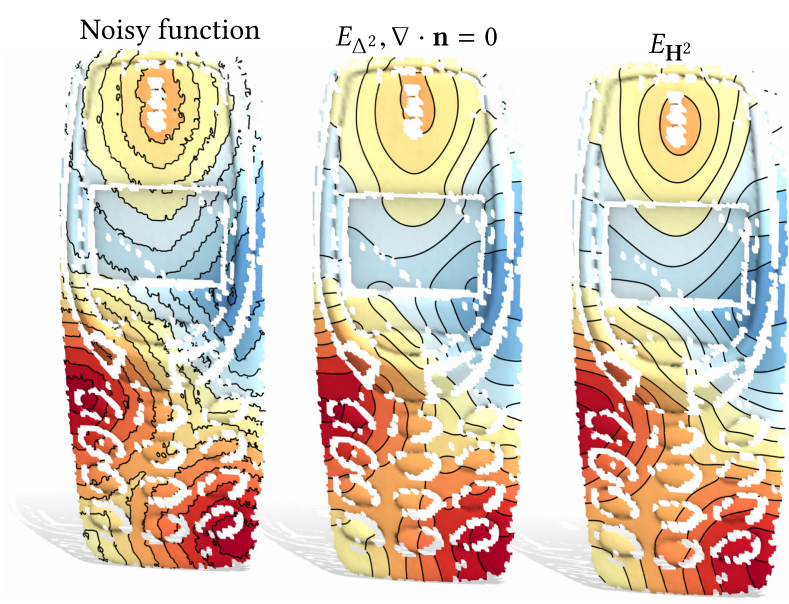

Fig. 13. Smoothing on a noisy domain. Compared to previous methods (left), the natural boundary conditions of $E_{\mathrm{H}^{2}}$ (right) better model ignoring the non-salient boundaries when smoothing data on a partial surface scan.

The biasing effect of these low-order conditions is apparent: the heavier the smoothing, the more the solution becomes constant near the boundary regardless of the data there. In contrast, smoothing with $E_{\mathbf{H}^{2}}$ allows the solution to vary near the boundary (see Figure 11).

In Figure 13, we smooth a noisy simulation of heat diffusion on a range scan of a Nokia cell phone (Godil et al. 2009). The abundance of free boundaries due to missing data highlights the effect of zero Neumann boundary conditions compared to the natural boundary conditions of $E_{\mathbf{H}^{2}}$.

The energies $E_{\Delta^{2}}$ and $E_{\mathrm{H}^{2}}$ measure the $L_{2}$-norm of the Laplacian/Hessian, respectively. Minimization of such energies prefers to distribute energy smoothly over the domain. In contrast, $L_{1}$ minimization prefers to concentrate high energy at sparse locations. At these locations, we see the behavior of natural boundary conditions of the smoothness energy. Effectively, they become boundaries between low-energy regions. In Figure 14, we smooth a toy function (a triangle wave plus noise). While $L_{2}$ smoothing $E_{\mathbf{H}^{2}}$ also rounds the peaks, $L_{1}$ smoothing $E_{\mathbf{H}^{1}}$ smooths away the noise but maintains the sharp creases.

In Figure 16, we smooth the noisy height data of a cathedral rooftop. Minimizing the $L_{1}$-norm of the Laplacian $\left(E_{\Delta^{1}}\right)$ concentrates energy at isolated points, producing a circus tent appearance. In contrast, minimizing the $L_{1}$-norm of the Hessian $\left(E_{\mathbf{H}^{1}}\right)$ concentrates energy along creases, producing a piecewise planar rooftop.

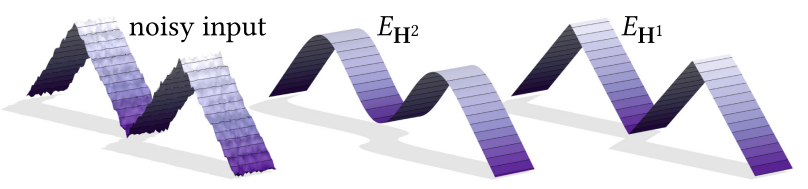

Fig. 14. Smoothing using the $L_{2}$-norm also removes salient creases. Minimizing the $L_{1}$-norm of the Hessian produces a piecewise-planar solution. 

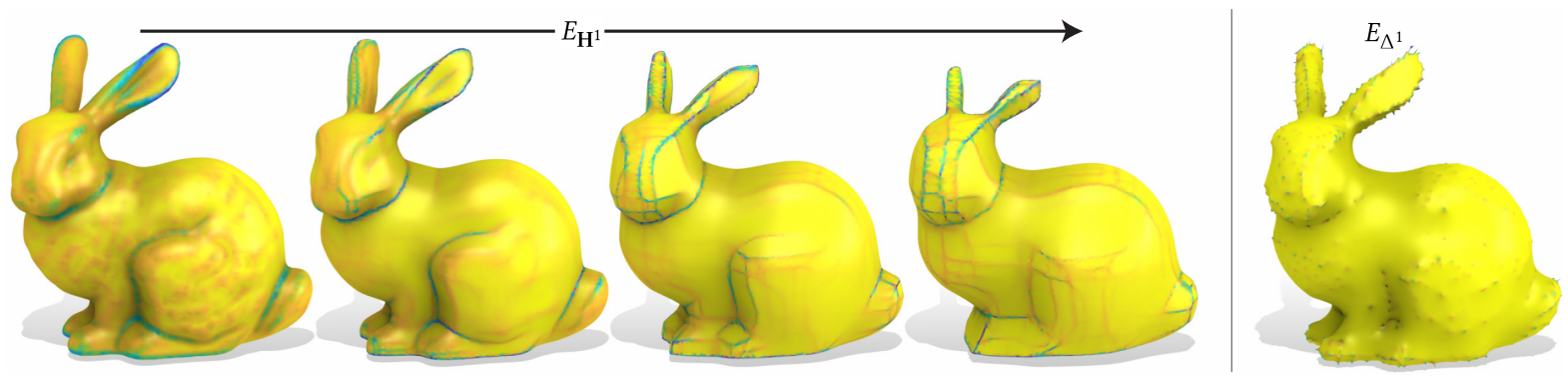

Fig. 15. The Bunny flows along $L_{1}$-minimization of the Hessian (local energy density in pseudocolor). Energy concentrates at creases and the bunny develops smooth, nearly flat regions. This minimization achieves crease-aware smoothing of surface geometry. Minimizing the $L_{1}$-norm of the Laplacian leads to energy concentration at points and a prickly appearance.

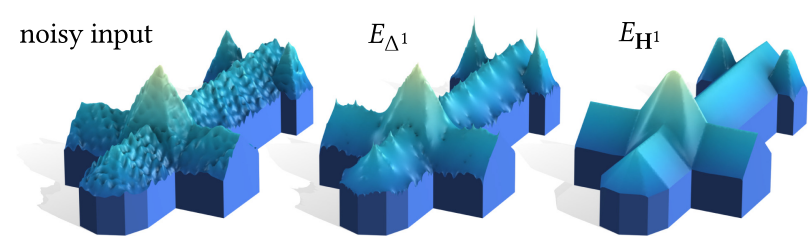

Fig. 16. Smoothing a cathedral roof. Unlike $L_{2}$ minimization, the behavior of $E_{\Delta^{1}}=\int|\Delta u| d A$ and $E_{\mathbf{H}^{1}}=\int\left|\mathbf{H}_{u}\right|_{F} d A$ are dramatically different in the interior.

Under the $L_{1}$-norm, the difference between minimizers does not rely on the presence of a boundary. Indeed, even for closed surfaces with no boundary, we see very different behavior. In Figure 15, we treat the surface's geometry as the input data $f$ and take smoothing steps where the data-versus-smoothness weight acts as an implicit time-step parameter controlling a geometric flow. The $E_{\mathbf{H}^{1}}$ flow of the Bunny forms 1D creases bounding smooth, neardevelopable (low Gaussian curvature) patches. This application is inspired by image smoothing with sparse norms (Xu et al. 2011) and, as such, the results are reminiscent of surface contrast enhancing methods (e.g., He and Schaefer (2013)). In contrast, the $E_{\Delta^{1}}$ flow quickly concentrates energy at isolated points suspended in a near-minimal (low mean curvature) surface.

\section{CONCLUSION AND FUTURE WORK}

Energies built with the Hessian rather than the Laplacian unlock high-order boundary conditions that are especially useful when boundaries are to be conceptually ignored during problem modeling.

In future work, we would like to investigate formulations of $E_{\mathbf{H}^{2}}$ for curved surfaces that account for the covariant derivative, as well as corresponding discretizations (see Section 5.3.1). For flat domains, it would be interesting to explore boundary-only discretizations (cf. Chen and Weber (2015)) or subspace deformation for solid objects. Initial derivations suggest that point constraints may not lead to smooth minimizers of second-order smoothness energies for solids. One possibility, however daunting, may be to consider the squared Frobenius norm of the three-tensor of third derivatives, an energy with only quadratic functions in its null space.
We hope that our article sheds new light on familiar problems and provides insights into the power of the natural boundary conditions of the squared Hessian energy for geometry processing problems. Many applications that are currently using the squared Laplacian energy with zero Neumann boundary conditions can potentially profit by trying the squared Hessian energy with natural boundary conditions alongside it.

\section{APPENDIXES}

\section{A FINITE DIFFERENCES}

To discretize $E_{\mathrm{H}^{2}}$ over a domain $\Omega$ embedded in a grid with $n$ total nodes and $\hat{n}$ interior nodes, we define a sparse matrix $\mathbf{H}=$ $\left[\begin{array}{lll}\mathbf{H}_{x x}^{\top} & \mathbf{H}_{y y}^{\top} & \sqrt{2} \mathbf{H}_{x y}^{\top}\end{array}\right]^{\top} \in \mathbb{R}^{3 \hat{n} \times n}$ so that each row of $\mathbf{H}_{x x}$ approximates the second derivative of the unknown function at the corresponding interior grid node: $\mathbf{H}_{x x}^{i} \mathbf{u} \approx \partial^{2} u\left(\mathbf{x}_{i}\right) / \partial x^{2}$ and analogously for rows in $\mathbf{H}_{y y}$ and $\mathbf{H}_{x y}$.

We use standard, second-order stencils for each term, i.e.:

$$
\begin{aligned}
\mathbf{H}_{x x}^{i} \mathbf{u} & =\frac{u_{i, j-1}-2 u_{i, j}+u_{i, j+1}}{h^{2}}, \\
\mathbf{H}_{y y}^{i} \mathbf{u} & =\frac{u_{i-1, j}-2 u_{i, j}+u_{i+1, j}}{h^{2}}, \\
\mathbf{H}_{x y}^{i} \mathbf{u}=\mathbf{H}_{y x}^{i} \mathbf{u} & =\frac{u_{i-1, j-1}+u_{i-1, j+1}-u_{i+1, j-1}+u_{i+1, j+1}}{4 h^{2}} .
\end{aligned}
$$

\section{B MIXED FINITE ELEMENTS}

Solving the piecewise-linear discretization of Equation (25) by differentiating with respect to all degrees of freedom, we have a system of linear equations in matrix form:

$$
\left(\begin{array}{cc}
\tilde{\mathbf{M}} & \mathrm{D}^{\mathrm{T}} \mathrm{AG} \\
\mathrm{G}^{\mathrm{T}} \mathrm{AD} & 0
\end{array}\right)\left(\begin{array}{c}
\mathbf{V}_{x x} \\
\mathbf{V}_{x y} \\
\mathbf{V}_{y x} \\
\mathbf{V}_{y y} \\
\mathbf{u}
\end{array}\right)=0,
$$

where $\tilde{\mathbf{M}} \in \mathbb{R}^{4|i| \times 4|i|}$ repeats the mass matrix $\mathbf{M}(i, i)$ along the diagonal, $\mathbf{A} \in \mathbb{R}^{2 m \times 2 m}$ is a diagonal matrix containing triangle areas, and $\mathbf{D} \in \mathbb{R}^{2 m \times 4|i|}$ computes the matrix divergence defined by

$$
\mathbf{D}=\left(\begin{array}{cccc}
\mathrm{G}(x, i) & \mathrm{G}(y, i) & 0 & 0 \\
0 & 0 & \mathrm{G}(x, i) & \mathrm{G}(y, i)
\end{array}\right),
$$

where $\mathrm{G} \in \mathbb{R}^{2 m \times n}$ is the usual gradient operator and $\mathrm{G}(x, i)$ selects the rows and columns corresponding to the $x$-components 
and interior vertices, respectively. Finally, if we use lumped mass matrices, we can efficiently condense this system and use it to define a discretization of our original energy:

$$
E_{\mathrm{H}^{2}}(u) \approx \mathbf{u}^{\top} \mathrm{G}^{\top} \mathrm{ADM} \tilde{M}^{-1} \mathrm{D}^{\top} \mathrm{AGu} .
$$

\section{C $L_{1}$ MINIMIZATION}

In the smooth setting, the $L^{1}$ Frobenius norm of the Hessian is

$$
\int_{\Omega}\left|\mathbf{H}_{u}\right|_{F} d A .
$$

This can be minimized by introducing an auxiliary matrixvalued variable equal to the element-wise absolute value of the Hessian $\mathrm{Y}=\left|\mathrm{H}_{u}\right|$ and solving the constrained optimization problem:

$$
\min _{u, \mathrm{Y}} \int_{\Omega} 1^{\mathrm{T}} \mathrm{Y} 1 d A,
$$

subject to $\mathrm{H}_{u} \leq \mathrm{Y}, \mathrm{H}_{u} \geq-\mathrm{Y}$, and $\mathrm{Y} \geq 0$,

where $\mathbf{1}$ is a vector of ones (with appropriate length).

Using our discrete matrices, this becomes a linear program

$$
\begin{aligned}
\min _{\mathbf{u}, \mathbf{y}} & \mathbf{1}^{T} \tilde{\mathbf{M y}}, \\
\text { subject to } & \mathbf{H u} \geq-\mathbf{y}, \\
& \mathbf{H u} \leq \mathbf{y}, \\
& \mathbf{y} \geq 0,
\end{aligned}
$$

where $\mathrm{y} \in \mathbb{R}^{4 \hat{n}}$ is a vectorization of per-vertex $2 \times 2$ matrices on a mesh with $\hat{n}$ interior vertices and $\mathrm{H}=\mathrm{D}^{\top} \mathrm{AG}$.

When combined with other quadratic energies such as a data term, this transforms into a quadratic program. We solve such problems with Mosek (Andersen and Andersen 2000).

\section{ACKNOWLEDGMENTS}

We would like to thank Ladislav Kavan, Denis Zorin, and Jinshuo Dong for early discussions; Yu Wang for sharing source code; Paul Kry for hosting the 2017 Bellairs workshop; and organizers of the Dagstuhl Seminar \#17232.

This work was funded in part by the Binational Science Foundation (US-Israel) Award 2012376, the National Science Foundation Award IIS-14-09286, the NSERC Discovery Grants (RGPIN-201705235 \& RGPAS-2017-507938), a Canada Research Chair award, the Connaught Fund, and a gift from Adobe Systems Inc.

\section{REFERENCES}

E. D. Andersen and K. D. Andersen. 2000. The MOSEK interior point optimizer for linear programming: An implementation of the homogeneous algorithm. In High Performance Optimization. Kluwer Academic Publishers, 197-232.

James Andrews, Pushkar Joshi, and Nathan Carr. 2011. A linear variational system for modeling from curves. Comput. Graph. Forum 30, 6, 1850-1861.

Omri Azencot, Maks Ovsjanikov, Frédéric Chazal, and Mirela Ben-Chen. 2015. Discrete derivatives of vector fields on surfaces-an operator approach. ACM Trans. Graph. 34, 3 (May 2015), Article 29, 13 pages. DOI:http://dx.doi.org/10.1145/ 2723158

Ilya Baran and Jovan Popović. 2007. Automatic rigging and animation of 3D characters. ACM Trans. Graph. 26, 3 (2007), 72:1-72:8.

Christopher Batty. 2010. Simulating Viscous Incompressible Fluids with Embedded Boundary Finite Difference Methods. Ph.D. Dissertation. University of British Columbia.

M. Belkin and P. Niyogi. 2004. Semi-supervised learning on Riemannian manifolds. Mach. Learn. 56, 1-3 (2004), 209-239.
Miklos Bergou, Max Wardetzky, David Harmon, Denis Zorin, and Eitan Grinspun. 2006. A quadratic bending model for inextensible surfaces. In Proceedings of the 4th Eurographics Symposium on Geometry Processing (SGP'06). 227-230.

Morten Bojsen-Hansen and Chris Wojtan. 2016. Generalized non-reflecting boundaries for fluid re-simulation. ACM Trans. Graph. 35, 4 (2016), Article 96.

F. L. Bookstein. 1989. Principal warps: Thin-plate splines and the decomposition of deformations. IEEE Trans. Pattern Anal. Mach. Intell. 11, 6, 567-585.

Mario Botsch and Leif Kobbelt. 2004. A remeshing approach to multiresolution modeling. In Proceedings of the 2nd Eurographics Symposium on Geometry Processing (SGP'04). 189-196.

M. Botsch and L. Kobbelt. 2005. Real-time shape editing using radial basis functions. Comp. Graph. Forum 24, 3 (2005), 611-621.

D. Braess. 2002. Finite Elements: Theory, Fast Solvers, and Applications in Solid Mechanics (2nd ed.; Vol. 13). Cambridge University Press.

D. Braess, A. S. Pechstein, and J. Schöberl. 2017. An equilibration based a posteriori error estimate for the biharmonic equation and two finite element methods. arXiv:1705.07607.

A. Bronstein, Y. Choukroun, R. Kimmel, and M. Sela. 2016. Consistent discretization and minimization of the 11 norm on manifolds. In Proceedings of the 4th International Conference on 3D Vision (3DV'16).

Chen Cao, Derek Bradley, Kun Zhou, and Thabo Beeler. 2015. Real-time high-fidelity facial performance capture. ACM Trans. Graph. 34, 4, Article 46.

Chen Cao, Yanlin Weng, Shun Zhou, Yiying Tong, and Kun Zhou. 2014. Facewarehouse: A 3D facial expression database for visual computing. IEEE Trans. Vis. Comput. Graph. 20, 3, 413-425.

Renjie Chen and Ofir Weber. 2015. Bounded distortion harmonic mappings in the plane. ACM Trans. Graph. 34, 4, Article 73.

Daniel Cohen-Steiner and Mathieu Desbrun. 2002. Hindsight: LSCM and DNCP Are One and the Same. Technical Report. INRIA-USC

M. I. Comodi. 1989. The Hellan-Herrmann-Johnson method: Some new error estimates and postprocessing. Math. Comput. 52, 185 (1989), 17-29.

Richard Courant and David Hilbert. 1924. Methoden der Mathematischen Physik I. Knight, Berlin, Germany.

Keenan Crane. 2009. A Cotangent Laplacian for Surfaces with Boundary. Technical Report. INRIA-USC.

Fernando de Goes, Beibei Liu, Max Budninskiy, Yiying Tong, and Mathieu Desbrun. 2014. Discrete 2-tensor fields on triangulations. Comp. Graph. Forum $33,5,13-24$.

Mathieu Desbrun, Mark Meyer, and Pierre Alliez. 2002. Intrinsic parameterizations of surface meshes. Comp. Graph. Forum 21, 3 (2002), 209-218.

Mathieu Desbrun, Mark Meyer, Peter Schröder, and Alan H. Barr. 1999. Implicit fairing of irregular meshes using diffusion and curvature flow. In Proceedings of the ACM SIGGRAPH Conference.

Stephan Didas and Joachim Weickert. 2004. Higher Order Variational Methods for Noise Removal in Signals and Images. Diploma Thesis. Saarland University, Saarbrücken.

Stephan Didas, Joachim Weickert, and Bernhard Burgeth. 2009. Properties of higher order nonlinear diffusion filtering. 7. Math. Imag. Vis. 35, 3 (2009), 208-226.

David L. Donoho and Carrie Grimes. 2003. Hessian eigenmaps: Locally linear embedding techniques for high-dimensional data. Proc. Natl. Acad. Sci. U S A 100, 10, 5591-5596.

Lawrence C. Evans. 1998. Partial Differential Equations. American Mathematical Society, Providence, RI.

Carlos A. Felippa. 2017. Advanced Finite Element Methods-Course Notes. Retrieved from https://www.colorado.edu/engineering/CAS/courses.d/AFEM.d/.

Mark Finch, John Snyder, and Hugues Hoppe. 2011. Freeform vector graphics with controlled thin-plate splines. ACM Trans. Graph. 30, 6, Article 166.

M. Fisher, P. Schröder, M. Desbrun, and H. Hoppe. 2007. Design of tangent vector fields. ACM Trans. Graph. 26, 3 (2007), Article 56.

Bengt Fornberg. 1988. Generation of finite difference formulas on arbitrarily spaced grids. Math. Comput. 51, 184, 699-706.

A. Garg, E. Grinspun, M. Wardetzky, and D. Zorin. 2007. Cubic shells. Proceedings of the 2007 ACM SIGGRAPH/Eurographics Symposium on Computer Animation. 91-98.

I. M. Gelfand and S. V. Fomin. 1963. Calculus of Variations. Prentice Hall.

T. G. Georgiev. 2004. Photoshop healing brush: A tool for seamless cloning. In Proceedings of the ECCV ACV Workshop. 1-8.

Mariano Giaquinta and Stefan Hildebrandt. 1996. Calculus of Variations I. Springer.

Y. I. Gingold, P. Davidson, J. Han, and D. Zorin. 2006. A direct texture placement and editing interface. In Proceedings of the 19th Annual ACM Symposium on User Interface Software and Technology. 23-32.

A. Godil, A. Axenopoulos, P. Daras, T. Furuya, and R. Ohbuchi. 2009. SHREC 2009: Shape retrieval contest of partial 3D models. In Proceedings of the Eurographics Workshop on 3D Object Retrieval.

Lei He and Scott Schaefer. 2013. Mesh denoising via L0 minimization. ACM Trans. Graph. 32, 4, Article 64.

Klaus Hildebrandt, Christian Schulz, Christoph Von Tycowicz, and Konrad Polthier. 2011. Interactive surface modeling using modal analysis. ACM Trans. Graph. 30 $5,119$. 
Ronald Hoppe, Dietrich Braess, and Christopher Linsenmann. 2016. A two-energies principle for the biharmonic equation and an a posteriori error estimator for an interior penalty discontinuous Galerkin approximation. ESAIM. Available at https: //www.esaim-m2an.org.

Jin Huang, Xiaohan Shi, Xinguo Liu, Kun Zhou, Li-Yi Wei, Shang-Hua Teng, Hujun Bao, Baining Guo, and Heung-Yeung Shum. 2006. Subspace gradient domain mesh deformation. ACM Trans. Graph. 25, 3 (2006), 1126-1134.

Alec Jacobson, Ilya Baran, Jovan Popović, and Olga Sorkine. 2011. Bounded biharmonic weights for real-time deformation. ACM Trans. Graph. 30, 4 (2011), Article 78.

A. Jacobson, Z. Deng, L. Kavan, and J. P. Lewis. 2014. Skinning: Real-time shape deformation. In ACM SIGGRAPH 2014 Courses.

Alec Jacobson, Daniele Panozzo, Christian Shuller, Olga Diamanti, Qingnan Zhou. Sebastian Koch, Jeremie Dumas, et al. 2017. Libigl: A Simple C++ Geometry Processing Library. Retrieved from http://libigl.github.io/libigl.

Alec Jacobson, Elif Tosun, Olga Sorkine, and Denis Zorin. 2010. Mixed finite elements for variational surface modeling. In Proceedings of the 8th Eurographics Symposium on Geometry Pressing (SGP'10).

Alec Jacobson, Tino Weinkauf, and Olga Sorkine. 2012. Smooth shape-aware functions with controlled extrema. In Proceedings of the 10th Eurographics Symposium on Geometry Processing (SGP'12).

Ben Jones, Nils Thuerey, Tamar Shinar, and Adam W. Bargteil. 2016. Example-based plastic deformation of rigid bodies. ACM Trans. Graph. 35, 4, Article 34.

Ladislav Kavan, Dan Gerszewski, Adam Bargteil, and Peter-Pike Sloan. 2011. Physicsinspired upsampling for cloth simulation in games. ACM Trans. Graph. 34, 4, Article 93.

Kwang I. Kim, Florian Steinke, and Matthias Hein. 2009. Semi-supervised regression using Hessian energy with an application to semi-supervised dimensionality reduction. In Advances in Neural Information Processing Systems.

Bishnu P. Lamichhane. 2011. A stabilized mixed finite element method for the biharmonic equation based on biorthogonal systems. f. Comp. Appl. Math. 235, 17, 5188-5197.

E. Landreneau and S. Schaefer. 2010. Poisson-based weight reduction of animated meshes. Comp. Graph. Forum 29, 6 (2010), 1945-1954.

Stamatios Lefkimmiatis, Aurélien Bourquard, and Michael Unser. 2012. Hessian-based norm regularization for image restoration with biomedical applications. IEEE Trans. Image Process. 21, 3 (2012), 983-995.

B. Lévy, S. Petitjean, N. Ray, and J. Maillot. 2002. Least squares conformal maps for automatic texture atlas generation. ACM Trans. Graph. 21, 3 (2002), 362-371.

W. L. Li. 2000. Free vibrations of beams with general boundary conditions. F. Sound Vib. 237, 4 (2000), 709-725.

Yaron Lipman, Raif Rustamov, and Thomas Funkhouser. 2010. Biharmonic distance. ACM Trans. Graph. 29, 3 (2010), Article 27.

Beibei Liu, Yiying Tong, Fernando De Goes, and Mathieu Desbrun. 2016. Discrete connection and covariant derivative for vector field analysis and design. ACM Trans. Graph. 35, 3, Article 23.

X. Y. Liu, C.-H. Lai, and K. A. Pericleous. 2015. A fourth-order partial differential equation denoising model with an adaptive relaxation method. Int. 7. Comput. Math 92, 3 (2015), 608-622.

Marius Lysaker, Arvid Lundervold, and Xue-Cheng Tai. 2003. Noise removal using fourth-order partial differential equation with applications to medical magnetic resonance images in space and time. IEEE Trans. Image Process. 12, 12, 1579-1590.

Marius Lysaker and Xue-Cheng Tai. 2006. Iterative image restoration combining total variation minimization and a second-order functional. Int. f. Comput. Vis. 66, 1, $5-18$.

Patrick Mullen, Yiying Tong, Pierre Alliez, and Mathieu Desbrun. 2008. Spectral conformal parameterization. In Proceedings of the 6th Eurographics Symposium on Geometry Processing (SGP'08).

Ulrich Pinkall and Konrad Polthier. 1993. Computing discrete minimal surfaces and their conjugates. Experiment. Math. 2, 1 (1993), 15-36.
Jean Marc Roth. 2009. Higher Order Anisotropic Smoothing of Images. Ph.D. Dissertation. Saarland University.

Leonid I. Rudin, Stanley Osher, and Emad Fatemi. 1992. Nonlinear total variation based noise removal algorithms. Phys. D: Nonlinear Phenom. 60, 1 (1992), 259-268.

Raif M. Rustamov. 2011. Multiscale Biharmonic Kernels. Retrieved from https://diglib. eg.org/handle/10.1111/v30i5pp1521-1531.

R. Scholz. 1978. A mixed method for 4th order problems using linear finite elements. RAIRO Anal. Numér. 1, 85-90.

Olga Sorkine, Yaron Lipman, Daniel Cohen-Or, Marc Alexa, Christian Rössl, and Hans-Peter Seidel. 2004. Laplacian surface editing. In Proceedings of the 2nd Eurographics Symposium on Geometry Processing (SGP'04). 179-188.

Boris Springborn, Peter Schröder, and Ulrich Pinkall. 2008. Conformal equivalence of triangle meshes. ACM Trans. Graph. 27, 3, Article 77.

Gabriele Steidl. 2006. A note on the dual treatment of higher-order regularization functionals. Computing 76, 1-2 (2006), 135-148.

Gabriele Steidl, Stephan Didas, and Julia Neumann. 2005. Relations between higher order TV regularization and support vector regression. In Proceedings of the International Conference on Scale-Space Theories in Computer Vision. 515-527.

Florian Steinke and Matthias Hein. 2009. Non-parametric regression between manifolds. In Advances in Neural Information Processing Systems. 1561-1568.

Daniel Sýkora, Ladislav Kavan, Martin Čadík, Ondřej Jamriška, Alec Jacobson, Brian Whited, Maryann Simmons, and Olga Sorkine-Hornung. 2014. Ink-and-ray: Basrelief meshes for adding global illumination effects to hand-drawn characters. ACM Trans. Graph. 33, 2, Article 16.

Demetri Terzopoulos. 1984. Multilevel reconstruction of visual surfaces: Variational principles and finite-element representations. In Multiresolution Image Processing and Analysis. Springer, 237-310.

Demetri Terzopoulos. 1988. The computation of visible-surface representations. IEEE Trans. Pattern Anal. Mach. Intell. 10, 4 (1988), 417-438.

E. Tosun, Y. I. Gingold, J. Reisman, and D. Zorin. 2007. Shape optimization using reflection lines. In Proceedings of the 5th Eurographics Symposium on Geometry Processing (SGP'07).

Yu Wang, Alec Jacobson, Jernej Barbič, and Ladislav Kavan. 2015. Linear subspace design for real-time shape deformation. ACM Trans. Graph. 34, 4, Article 57.

Yu Wang, Alec Jacobson, Jernej Barbič, and Ladislav Kavan. 2017. Error in "Linear Subspace Design for Real-Time Shape Deformation”. Technical Report.

Ofir Weber, Roi Poranne, and Craig Gotsman. 2012. Biharmonic coordinates. In Comput. Graph. Forum, 31, 2409-2422.

Tino Weinkauf, Yotam Gingold, and Olga Sorkine. 2010. Topology-based smoothing of 2D scalar fields with $C^{1}$-continuity. Comput. Graph. Forum 29, 3 (2010), 12211230 .

Li Xu, Cewu Lu, Yi Xu, and Jiaya Jia. 2011. Image smoothing via L0 gradient minimization. ACM Trans. Graph. 30, 6, Article 174

Y.-L. You and M. Kaveh. 2000. Fourth-order partial differential equations for noise removal. IEEE Trans. Image Process. 9, 10 (2000), 1723-1730.

Jing Yuan, Christoph Schnörr, and Gabriele Steidl. 2009. Total-variation based piecewise affine regularization. In Proceedings of the International Conference on Scale Space and Variational Methods in Computer Vision. 552-564.

Hao Zhang, Oliver van Kaick, and Ramsay Dyer. 2007. Spectral methods for mesh processing and analysis. In Proceedings of the Eurographics State-of-the-Art Report. $1-22$.

Kun Zhou, Jin Huang, John Snyder, Xinguo Liu, Hujun Bao, Baining Guo, and HeungYeung Shum. 2005. Large mesh deformation using the volumetric graph Laplacian. ACM Trans. Graph, 24, 3 (2005), 496-503.

Kun Zhou, Weiwei Xu, Yiying Tong, and Mathieu Desbrun. 2010. Deformation transfer to multi-component objects. Comp. Graph. Forum 29, 2 (2010), 319-325.

Received October 2017; revised December 2017; accepted February 2018 